\title{
Prevalence of intestinal schistosomiasis and soil transmitted helminthiasis among primary school children in Umolante district, South Ethiopia
}

\author{
Megbaru Alemu ${ }^{1,}$, , Asrat Hailu², Gessessew Bugssa ${ }^{1}$ \\ ${ }^{1}$ Institute of Biomedical Sciences, College of Health Sciences, Mekelle University, Mekelle, Ethiopia \\ ${ }^{2}$ Department of Micrbiology, Immunology and Parasitoloogy, Medical faculty, Addis Ababa University, Addis Ababa, Ethiopia \\ Email address: \\ megibgesep@gmail.com(M. Alemu), hailu_a2004@yahoo.com (A. Hailu),bugssag@gmail.com (G. Bugssa)
}

\section{To cite this article:}

Megbaru Alemu, Asrat Hailu, Gessessew Bugssa. Prevalence of Intestinal Schistosomiasis and Soil Transmitted Helminthiasis among Primary School Children in Umolante District, South Ethiopia. Clinical Medicine Research. Vol. 3, No. 6, 2014, pp. 174-180. doi: $10.11648 /$ j.cmr.20140306.14

\begin{abstract}
Introduction: Intestinal Schistosomiasis and Soil transmitted helminthic infections (STIs) are among the major public health problems in the world, especially in Subsaharan Africa. Objective: To determine the prevalence of intestinal schistosomiais and soil transmitted helminthic infections and associated factors among Umolante primary school children, South Ethiopia. Methods: A cross sectional study was conducted among Primary School Children in Umolante Destrict, South Ethiopia. A total of 405 school children were selected at random. For each selected subject interview and stool examination was done. Interviews were used to identify the risk factors. Stool specimens were examined using the Kato-Katz technique. Data was entered and analyzed using SPSS version 16.0 statistical packages software. Results: The overall prevalence of intestinal helminthic infection was 26.9\%. The predominant parasites were hookworm 59(14.6\%) and S.mansoni 51(12.6\%). Prevalence of S.mansoni infection was significantly higher in males $(\mathrm{p}=0.006)$ whereas hookworm infection was significantly higher in females $(\mathrm{P}=0.015)$. Other helminthes found were E.vermicularis $(1 \%)$, whipworm $(1.5 \%)$, tapeworm $(1.5 \%)$ and A.lumbricoides $(0.5 \%)$. The highest prevalence of S.mansoni was reported in the age group of $10-14$ years $(17.8 \%)$ followed by the age group 15 and above $(11.6 \%)$ and the difference was statistically significant $(\mathrm{P}=0.02)$. Geometric mean intensity was also highest in the same age group (245 eggs per gram). The overall prevalence of infection was $7.7 \%$ for girls and $17.1 \%$ for boys and the difference was statistically significant $(\mathrm{p}=0.006)$. Bathing practice in the nearby stream was significantly associated with S.mansoni infection (OR, 3.4, 95.0\% C.I., 1.5-5.3, $\mathrm{P}=0.03$ ). Conclusion and recommendation: On the basis of these results, it can be concluded that intestinal helminthic infections are important health problems among school children. Hence, integrated control programme including periodic deworming, shoes wearing, improving sanitation, provision of safe water supply are needed to have a lasting impact on transmission these diseases.
\end{abstract}

Keywords: Prevalence, S.mansoni, Soil Transmitted Helminthes, Children, Umolante

\section{Introduction}

Intestinal schistosomiasis and soil transmitted helminthic infections (STIs) are among the major public health problems in the world, especially in Subsaharan Africa [1,2]. Current estimates indicate that an estimated 4.5 billion individuals are at risk of STH infections and the global estimate of number of cases of Ascaris lumbricoides is 807 million, Trichuris trichiura 604 million, Hookworm (Necator americanus; Anclystoma duodenale) 576 million, Schistosomiasis (Schistosoma haematobium, S.mansoni and
S.japonicum) 207 million [3-5].

The soil-transmitted helminthes are one of the world's most important causes of physical and intellectual growth retardation in children leading to attention deficits, learning disabilities, school absenteeism and higher dropout rates [6]. Schistosomiasis also remains one of the most prevalent parasitic diseases in the world. It is endemic in 76 countries and continues to be public health concern in developing countries. Approximately $80 \%$ of the 200 million people infected world-wide live in sub-Saharan Africa where S.mansoni and S.haematobium are widespread [7]. 
In Ethiopia, like in other developing countries, intestinal parasitic infections are widely spread. A considerable proportion of annual visits at outpatient services of the health institutions are due to such infections [8]. Many reports illustrated that A.lumbricoides is the most prevalent intestinal parasite in different communities usually occurring together with trichuris infections $[9,10]$. Hookworm infection, strongyloidiasis and enterobiasis are also public health problem though the magnitude is lesser compared to ascariasis. The prevalence of taeniasis alone ranges from 1$48 \%$ and the infection rate with Hymenolopis nana is 3- $61 \%$ $[11,12,13]$. Intestinal Schistosomiasis is widely spread in the country. Rapid spread of the disease also appears to have been facilitated in areas which were originally non-endemic as a result of the initiation of water-based development schemes [14].

Approximately, a couple of decades have elapsed since the last epidemiological study was conducted in the area by Woldemichael et al [8] and the current status of the disease is not known. Hence, the objective of this study was to determine the current prevalence of intestinal helminthic infections, identify associated risk factors and assess the intensity of infections among school children of Umolante.

\section{Materials and Methods}

\subsection{Study Area}

The study was conducted among Primary School Children of Umolante, Gamogofa Zone, South Ethiopia. Umolante district is found in the South Nations, Nationalities and Peoples Region (SNNPR) located at $491 \mathrm{kms}$ south of Addis Ababa, the capital of the country. In Umolante district, there were six water schemes which were no more functional since the year 2008. Pipe water transported through carts from Lante (the nearby district) is the only water source for drinking and cooking. Besides, the district has a small stream called Basso which passes near the school where children swim, bath their body, and wash clothes and dishes.

Study design: A cross sectional study design was employed to study the prevalence of intestinal schistosomiasis and soil transmitted helminthes (STH) among Primary School Children in Umolante District, South Ethiopia in 2012.

\subsection{Study Population}

The study population included all school children attending from grade one to eight in Umolante primary school on that year.

Inclusion criteria: Students who were volunteer were eligible to participate.

Exclusion Criteria: Students with mental problem and non volunteers were excluded from the study.

\subsection{Sample Size and Sampling Technique}

Sample size was determined using single population proportion estimate considering the level of significances at $5 \%$ and the prevalence of intestinal helminthiasis to be $60 \%$ from a previous study conducted elsewhere [8]. Using the formula $\left(n=z^{2} p(1-p) / d^{2}\right.$, where $n=$ sample size, $z=z$ statistic for a level of confidence $(\mathrm{z}=1.96$ at $95 \% \mathrm{CI}), \mathrm{p}=$ expected prevalence or proportion $(\mathrm{p}=0.6), \mathrm{d}=$ precision (if $5 \%, \mathrm{~d}=$ 0.05 ). Considering $10 \%$ non response rate, the final sample size was calculated to be 405 .

Multistage sampling method was used to select the study subjects. Classes were first selected by simple random sampling technique and then the sampled children were selected by systematic random sampling using class roster (students list) as a sampling frame, and proportional allocation was employed to select students from each selected section.

\subsection{Data Collection Techniques}

Questionnaire: A pre-tested questionnaire based on known risk factors was developed and modified. The interview included information such as age, sex, source of drinking water, existence of latrines in their homes, and yes or no choice questions for domestic activities in the nearby stream such as swimming, bathing and dishwashing and their habits of latrine usage.

Parasitological Examination: After being interviewed, the students were then supplied with labeled stool containers with tight covers bearing serial numbers of the subjects and were requested to bring about $5 \mathrm{~g}$ of stool. All the stool samples were received at the spot at an organized central place. The specimens were processed for microscopic examination using Kato-Katz technique [15] (two slides per stool specimen) employing a template delivering a plug of $41.7 \mathrm{mg}$ of stool and examined by experienced technician under 10X and 40X objective lens. Averages of egg count on pair of slides per sample were taken and conversions into eggs per gram (epg) of stool were done by a factor of 24 .

Finally, quantification of egg load for evaluation of intensity of infection was performed. Intensity of infection was estimated from the number of eggs per gram (epg) of faeces. Based on egg counts, cut-off values for classification of intensity of infection were used. Intensity of S.mansoni was classified into: light infection (1-99 epg), moderate (100399 epg) and heavy (greater than 400 epg). Similarly, the classification for A.lumbricoides: light infection (1-4999 epg), moderate (5000-49999 epg) and heavy (greater than 50,000 epg); T.trichiura: light infection (1-999epg), moderate (1000-9999 epg) and heavy (greater than 10,000 epg) and for hookworm: light infection (1-1999epg), moderate (20003999 epg) and heavy (greater than 4,000 epg) was made according to the standard [16].

Data management and quality control: Standard Operating procedure (SOP) was sternly followed during the course of preparation and examination of Kato thick smears.

Data processing and analysis: Data were coded, entered into computer and cleaned before and during data processing using EPI INFO version 6 and exported to SPSS version 16.0 software package for statistical analysis. Different variables were described and characterized by frequency distribution. 
Prevalence and intensity of S.mansoni and STH infection were reported in percent and mean egg count, respectively. The association was statistically tested using univariate and multiviarate regression analysis. The magnitude of association was measured by odds ratio at $95 \% \mathrm{CI}$ : P-value below 0.05 was considered as statistically significant.

Ethical clearance: Ethical clearance to conduct the research was obtained from Department of Microbiology, Immunology and Parasitology and Institutional Review Board of Faculty of Medicine, Addis Ababa University via submission of the study protocol before the study commences. Prior to data collection, the objectives of the study were clearly discussed with the school community. Written informed consent was obtained from the parents and teachers of the students before stool samples collection.

All children who were tested positive for S.mansoni were treated with a single dose of praziquantel at $40 \mathrm{mg} / \mathrm{Kg}$ body weight and children tested positive for A.lumbrcoides, T. trichiura and hookworm were treated with standard regiment albenidazol 400mg tablet.

\section{Results}

\subsection{Socio Demography of Study Subjects}

A total of 405 students participated in the study yielding response rate of $100 \%$. Of the study participants, females constitute $47.9 \%$ of the study subjects giving a sex ratio of 1.09:1. The mean (SD) age of the participants was $11.2 \pm 3.4$ years ranging from 5 to 18 years. Majority $(51.1 \%)$ of the study participants fall within the age category $10-14$ years followed by $129(31.9 \%$ ) falling in the age group of 5-9 years.

\subsection{Prevalence of Parasites}

A total of six parasite species were identified, namely: $S$. mansoni, Hookworm species, Taenia species, E.vermicularis, T.trichuria and A.lumbricodes. Among the 405 study participants, $109(26.9 \%)$ of them harboured at least one helminth parasite out of which hookworms and S.mansoni accounted for most of the infections with prevalence rate of $14.6 \%$ and $12.6 \%$, respectively. A total of $18(4.4 \%)$ coinfections were found, hookworm and S.mansoni coinfection being the highest (3.2\%) (Table 1).

The highest proportion of parasites was reported among the age group of 10-14 years old subjects (31.4\%) followed by the age groups $\geq 15$ and $5-9$ years with infection rates of $27.5 \%$ and $19.4 \%$, respectively. The overall prevalence was slightly higher among boys than girls (27.5\% vs. $26.3 \%)$. However, the difference was not statistically significant. On the other hand, S.mansoni infection was found significantly higher in males $[\mathrm{p}=0.006$, OR 2.5, CI 1.3-2.5]. Besides, hookworm infection was also found significantly higher among females $[p=0.015]$. Even though it was not statistically significantly, an infection of Taenia species was higher among boys whereas trichuriasis and entrobiasis were a bit higher among the female participants. A.lumbricoides showed no gender difference in prevalence [ $\mathrm{p}>0.05]($ Table 1$)$.

Table 1. Prevalence of S.mansoni and STH infections by sex among Umolante primary School Children, South Ethiopia.

\begin{tabular}{lllll}
\hline \multirow{2}{*}{ Parasite species } & Sex & & & \\
\cline { 2 - 4 } & Male (n=211) & Female (n=194) & Total (n=405) & P-value \\
\hline Single infection(overall) & $58(27.5 \%)$ & $51(26.3 \%)$ & $109(26.7 \%)$ & 0.77 \\
Hookworm only & $17(8.1 \%)$ & $28(14.4 \%)$ & $45(11.1 \%)$ & 0.015 \\
S.mansoni only & $30(14.2 \%)$ & $6(3.1 \%)$ & $36(8.9 \%)$ & 0.006 \\
Taenia sp.only & $3(1.4 \%)$ & $1(0.5 \%)$ & $4(1 \%)$ & 0.38 \\
E.vermicularis only & - & $3(1.5 \%)$ & $3(0.7 \%)$ & 0.96 \\
T.trichuria only & - & $2(1 \%)$ & $2(0.5 \%)$ & 0.95 \\
A.lumbricoides only & $1(0.5 \%)$ & $1(0.5 \%)$ & $2(0.5 \%)$ & 0.87 \\
Double infection(overall) & $7(3.3 \%$ & $11(5.7 \%)$ & $18(4.4 \%)$ & 0.26 \\
Hookworm and S.mansoni & $5(2.4 \%$ & $8(4.1 \%)$ & $13(3.2 \%)$ & 0.32 \\
S.mansoni and T.trichuria & - & $1(0.5 \%)$ & $1(0.2 \%)$ & 0.99 \\
S.mansoni and Taenia sp. & $1(0.5 \%)$ & - & $1(0.2 \%)$ & 0.99 \\
Hookworm and T.trichuria & - & $1(0.5 \%)$ & $1(0.2 \%)$ & 0.99 \\
E.vermicularis and T.trichuria & - & $1(0.5 \%)$ & $1(0.2 \%)$ & 0.99 \\
Taenia sp. and T.trichuria & $1(0.5 \%)$ & - & $1(0.2 \%)$ & 0.99 \\
\hline
\end{tabular}

*Binary logistic regression was used to calculate p- value; sp: species

Of the study participants, $109(26.7 \%)$ had single infections whereas $18(4.4 \%)$ of the study participants had double infections (Table 1). Meanwhile, the prevalence of any parasitic coinfection was also determined among the different age groups of the study subjects.

Of the study subjects, 65 (31.4\%) which belong to the age group 10-14 years had single infections (Table 1) whereas children falling to the age group 15 and above years had slightly higher $(5.8 \%)$ double infection than their counterparts (Table 2). S.mansoni and hookworm confection was found to be higher with prevalence rate of $13(3.2 \%)$. However, there was not any significant association for each of parasitic coinfections among the different age groups of the study subjects $(\mathrm{P}>0.05)$ (Table 2$)$. 
Table 2. Prevalence of single and multiple helminthic infections among different age groups Umolante Primary School children, South Ethiopia.

\begin{tabular}{|c|c|c|c|c|}
\hline \multirow{2}{*}{ Parasite species } & \multicolumn{3}{|c|}{ Age groups (years) } & \multirow{2}{*}{ p-value } \\
\hline & $5-9(n=129)$ & $10-14(n=207)$ & $15+(n=69)$ & \\
\hline Any single infection & $25(19.4 \%)$ & $65(31.4 \%)$ & $19(27.5 \%)$ & 0.10 \\
\hline Hookworm only & $14(10.9 \%)$ & $26(10.6 \%)$ & $9(13 \%)$ & 0.70 \\
\hline S.mansoni only & $2(1.6 \%)$ & $28(13.5 \%)$ & $6(8.7 \%)$ & 0.02 \\
\hline Taenia $s p$.only & & $4(1.9 \%)$ & - & 0.66 \\
\hline E.vermicularis only & $2(1.6 \%)$ & $1(0.5 \%)$ & _- & 0.22 \\
\hline T.trichuria only & $2(1.6 \%)$ & - & - & 0.99 \\
\hline A.lumbricoides only & - & $2(1 \%)$ & _ & 0.76 \\
\hline Any double infection & $5(3.9 \%)$ & $9(4.3 \%)$ & $4(5.8 \%)$ & 0.56 \\
\hline Hookworm and S.mansoni & $4(3.1 \%)$ & $8(3.9 \%)$ & $1(1.4 \%)$ & 0.66 \\
\hline S.mansoni and T.trichuria & - & $1(0.5 \%)$ & - & 0.83 \\
\hline S.mansoni and Taenia $s p$. & - & - & $1(1.4 \%)$ & 0.12 \\
\hline Hook worm and T.trichuria & $1(0.8 \%)$ & 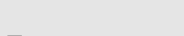 & 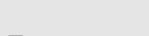 & 0.90 \\
\hline E.vermicularis and T.trichuria & _ & _- & $1(1.4 \%)$ & 0.95 \\
\hline Taenia $s p$. and T.trichuria & & & $1(1.4 \%)$ & 0.97 \\
\hline
\end{tabular}

*Binary logistic regression was used to calculate p- value, 15+: 15 years and above, sp: species

Moreover, the intensity of each parasite among the study subjects was also determined. Accordingly, the intensities of all helminthic infections except for S.mansoni were found to be light. The infection of T.trichuria was found to be light (1999) in all age groups whereas 2 cases of E.vermicularis and one case of A.lumbricoides infection were found to be moderately infected, respectively.
As shown in figure 1, prevalence of hookworm infection showed decline until the age group of 10-14 years and then showed increasing trend thereafter. However, prevalence of S.mansoni infection increased with age until the age of 10-14 years and then declined thereafter. Intensity of S.mansoni infection peaked in the age group of 10-14 years.

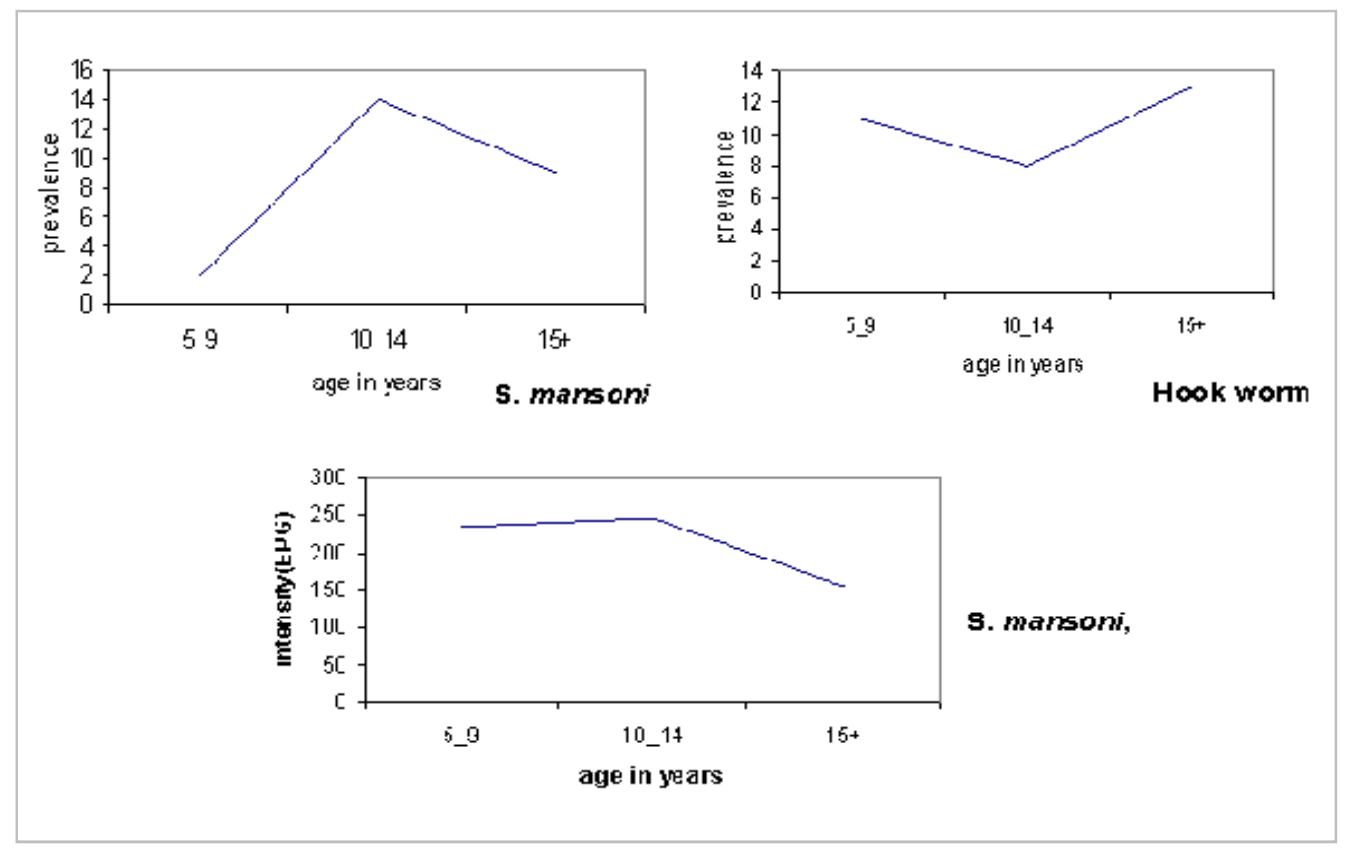

Figure 1. Prevalence and intensity curves of S.mansoni and Hookworm infection by age among positive children of Umolante Primary School, South Ethiopia.

Prevalence and intensity of S.mansoni infection: The overall prevalence of S.mansoni infection was 51(12.6\%) with parasitic load ranging from 24-960 eggs per gram of stool. S.mansoni infections were predominantly light; $70.5 \% \%$ light ( $<100 \mathrm{epg}), 21.6 \%$ moderate (100 - $400 \mathrm{epg})$ and $7.8 \%$ heavy ( $>400$ epg) infections. The highest prevalence was reported in the age group of $10-14$ years $(17.8 \%)$ followed by the age group 15 and above years $(11.6 \%)$ and $5-9(4.7 \%)$ and the difference was statistically significant $(\mathrm{P}=0.02)$. Geometric mean intensity was also highest in the age group 10-14 years (245epg). The overall prevalence of infection was $7.7 \%$ for girls and $17.1 \%$ for boys and the difference was statistically significant $(\mathrm{p}=0.006)$. The mean intensity of infection was also higher in boys (239 epg) compared to their 
counter parts (184 epg) (Table 3).

Table 3. Prevalence and intensity of S.mansoni infection among children of Umolante Primary school, South Ethiopia.

\begin{tabular}{lllllllll}
\hline $\begin{array}{l}\text { Age } \\
\text { (Years) }\end{array}$ & №. (\%) Males & & No.. (\%) females & & \multicolumn{2}{c}{ Over all, № (\%) } & \multicolumn{2}{c}{ Mean intensity (EPG) } \\
\cline { 2 - 9 } & Examined & + ves & Examined & + +ves & Examined & +ves & males & females \\
\hline $5-9$ & 65 & $2(3.1)$ & 64 & $4(6.3)$ & 129 & $6(4.7)$ & 248 & 222 \\
$10-14$ & 102 & $26(25.5)$ & 105 & $11(10.5)$ & 207 & $37(17.9)$ & 280 & 209 \\
+15 & 44 & $8(18.2)$ & 25 & $0(0)$ & 69 & $8(11.6)$ & 188 & 120 \\
Total & 211 & $36(17.1)$ & 194 & $15(7.7)$ & 405 & $51(12.6)$ & 239 & 184 \\
\hline
\end{tabular}

№ : Number; EPG: eggs per gram; +ves: positive for S.mansoni

Water contact behaviours and local risk factors: Domestic activities accounted for the majority of reported water contacts. Majority (64.3\%) of subjects interviewed reported that they bathed in the nearby stream. Similarly, about $60 \%$ of the respondents came into contact with water as the result of cloth and dishwashing. Besides, $15 \%$ of the respondents swam or played in the stream, and about $77 \%$ of the respondents reported that the water for drinking and cooking was collected exclusively from pipes and the rest was collected from both pipe and streams.

This study also showed that boys had more water contact habits like bathing, swimming, cloth and dishwashing in the nearby stream (Baso river). Accordingly, out of the 261 subjects who reported that they bathed in the stream, boys accounted for $65 \%$. With regard to swimming, the males accounted for $85 \%$ whereas nearly equal proportion of both sexes reported that they came into contact with stream water while washing clothes and dishes. Logistic regression results pointed out significant association between S.mansoni infection and bathing behaviours in the stream (OR, 3.4, 95.0\% C.I., 1.5-5.3, $\mathrm{P}=0.03$ ). Higher risks of infection were also showed for water contacts during swimming and dishwashing even though the difference was not significant. The study also revealed that subjects mostly affected with S.mansoni were those who used to drink from both pipe and stream water $(20 \%)$ compared to those who exclusively drunk pipe water.

All $(100 \%)$ of the students' families had latrine out of which $85 \%$ of them used their latrines, $13.3 \%$ of the respondents reported that they used to defecate in open fields 'sometimes', and the remaining (2.5\%) defecate under bushes. Three hundred five (75.3\%) of the children regularly practiced hand washing before meals whereas $323(79.6 \%)$ trimmed their fingernails.

Analysis of local risk factors showed, of the respondents who used latrine 'always', 94(27.6\%) of them had intestinal helminthic infection while $12(22.2 \%)$ of the participants who used toile 'some times' were positive for at least one intestinal helminth. On the other hand, those who did not use latrine at all seemed to be more infected (30\%) with intestinal helminth though the difference is not statistically significant. Though the difference is not significant, higher rates of E.vermicularis infection were observed among children who didn't wash their hands regularly before meals. Similarly, the prevalence of E.vermicularis was relatively higher among children who didn't trim their finger nails. Furthermore, the prevalence of other helminthes among children who washed their hands regularly and trimmed their fingers was not different from children who didn't ( $p>0.05$ ). Two hundred eighty five (70.4\%) of the children had shoes that gave full protection. The difference in the prevalence rate of hookworm infection between those with and without fully protective shoes was not statistically significant $(p>0.05)$.

\section{Discussion}

Epidemiological study on the prevalence of intestinal parasites in different regions/localities is a primary objective to identify high-risk communities and formulate appropriate intervention [17]. In line with this view, the present study attempted to assess the prevalence of different intestinal helminthic infections in school children in Umolante district, South Ethiopia. The results of the study showed the occurrence of several intestinal helminthes is of public health importance among the schoolchildren.

The overall prevalence of geohelminthes and S.mansoni infection in this study was found to be $26.9 \%$. This was comparable with findings from Tigray region and eastern Ethiopia which revealed overall prevalence of $28.6 \%$ and $27.2 \%$, respectively $[9,11]$. In contrast, the finding of this study was higher than reports from Kenya $[17,18]$ and Sudan [5] that revealed prevalence of $12.9 \%$ and $12.3 \%$, respectively. However, it was much lower than the previous study conducted in the same area which showed an infection rate of $60 \%$ [8]. The differences in findings among the studies can be explained by variations in geography, socioeconomic and hygienic conditions of the population under consideration.

In the present study, hookworm was found to be the dominant STH (14.6\%). This is consistent with previous study conducted in the area and preponderance of hookworm infection could be attributed mainly to the sustainability of microclimate, soil type and humid environment favourable for parasite development [8]. Another study conducted in the eastern part of the country showed lower rates of hookworm infection (6.7\%) [14]. In contrary to the present finding, higher rates of hookworm were also reported near lake Awassa (62.5\%), Langano (64.7\%) and South west Ethiopia $(40.8 \%)[10,12,19]$. The present study also revealed that 
hook worm infection was significantly higher in girls $(19.5 \%)$ compared to boys $(10.2 \%)$. This study goes in agreement with studies conducted elsewhere where hookworm infection was lower among boys [11, 20]. Contrary to this result, higher prevalence of hook worm was recorded for boys in southwest Ethiopia [12].

The overall prevalence of infections of Taenia $s p$, T.trichuria, E.vermicularis and A.lumbricoides were generally low. This is similar to other studies conducted elsewhere [5, 20-24]. The findings of the current study also depicted that intestinal helminthic infections are more prevalent in the age groups 10-14 years in the study area. This is an indication that younger children are more exposed since they usually play in the open fields [9] and they frequently involve themselves fully in activities that bring them in contact with the source of infection [25]. Another study conducted elsewhere also showed that highest infection rates among the age groups 5-14 years [23]. Results from rural Brazil also confirmed infection rates peaked in the age groups 11-15 years [21]. However, contrary to this finding, intestinal helminthic infections were found to be higher among the age groups $0-5$ years as indicated by studies from Nigeria [22].

The prevalence of S.mansoni infection in this study was $12.6 \%$ which is higher than the previous reports conducted in the same area and elsewhere [5, 8, 11, 25]. Generally, increased prevalence of S.mansoni is being reported in many studies [21, 26]. These differences in the prevalence of S.mansoni may be ascribed to altitude differences, availability of streams/rivers, ponds and playing habits of school children. Meanwhile, this study revealed that S.mansoni infections were predominantly light (70.5\%) followed by moderate $(21.6 \%)$ and heavy $(7.8 \%)$ infections in the study population. This finding is consistent with the findings from Uganda which showed $62.7 \%, 27.4 \%$ and $9.8 \%$ light, moderate and heavy infections, respectively [27]. As it is indicated in this study, the prevalence of S.mansoni was found to be higher among the boys than their counterparts. This study goes in agreement with the previous study done in the same area (8). This study also showed that mean intensity of infection was higher in boys compared to girls which is consistent with the findings conducted in some parts of the country and elsewhere in Africa [11, 19, 25]. These studies connote that higher infection rates and infection intensities in boys. The existence of more outdoor activities among boys than girls could be one of the reasons for this finding. This observation is supported by the fact that few of the girls had any history of playing or swimming in local water sources.

Moreover, in the present study highest prevalence and intensity of S.mansoni infection was found in the age group 10-14 years. These findings are similar with studies conducted in Jiga (North western Ethiopia) [26] and Nigeria [22]. Findings from Ghana also revealed the highest prevalence of infection in the age groups 6-11 and 12-17 years [28]. However, findings from Uganda disclosed the highest geometric mean intensity of S.mansoni infection in the age group 9-11 years [27].

\section{Conclusion and Recommendations}

S.mansoni and intestinal helminthic infections are becoming main health problems among school children. Children having more contact with stream as a result of domestic activities such as bathing, dish and cloth washing were more infected. Male sex and bathing in a stream were strongly associated with S.mansoni infection whereas hook worm infection was significantly higher in girls. Hence, high prevalence of intestinal helminthiasis in the study area shows the need of integrated control programme including periodic deworming, wearing shoes, improving sanitation, provision of safe water supply and latrines to reduce human-water contact accompanied by appropriate health education and other environmental measures to have a lasting impact on transmission of these diseases.

\section{References}

[1] WHO (World Health Organization). Informal consultation on intestinal helminthes infection. Geneva, Switzerland: World Health Organization, 1990.

[2] Alemu A, Atnafu A, Addis Z, Shiferaw Y, Teklu T, Mathewos B, Birhan W, Gebretsadik S, Gelaw B, Soil transmitted helminthes and schistosoma mansoni infections among school children in zarima town, northwest Ethiopia. BMC Infectious Diseases. 2011; 11:189.

[3] . Bethony J, Brooker S, Albonico M, Geiger SM, Loukas A, Diemert D, Hotez PJ. Soil transmitted helminth infections: ascariasis, trichuriasis, and hookworm. Lancet. 2006; 367: $1521-1532$.

[4] Hotez PJ, Brindley P, Bethony J, King CH, Pearce EJ, Jacobson J. Helminthic infections: the great neglected tropical diseases. Journal of Clinical Investigation. 2008; 118:1311-1321.

[5] Magambo JK, Zeyhle E, Wachira TM. Prevalence of Intestinal parasites among children in southern Sudan. East African Medical Journal. 1998; 75:288-90.

[6] Stephenson L, Latham M, Adams E, Kinoti S, Pertet A. Physical fitness, growth and appetite of Kenyan school boys with hookworm, Trichuris trichiura and Ascaris lumbricoides infections are improved four months after a single dose of albendazole. Journal of Nutrition. 1993; 123:1036-1046.

[7] Davis A, Cook C, Zumla A. Schistosomiasis: Manson's Tropical Diseases. London: Elsevier Science. 2003; 21: 14311469.

[8] Woldemichael T, Endeshaw T, Shibre T, Gebre T, Haddis M, Tilahun $\mathrm{D}$, et al. Intestinal parasitic infections in western Abaya with special reference to Schistosoma mansoni. Ethiopian Journal of Health Development. 1999; 13:25-26.

[9] Tadesse D, Tsehaye A. Impact of irrigation on the prevalence of intestinal parasite infections with emphasis on schistosomiasis in Hintallo-Wejerat, North Ethiopia. Ethiopian Journal of Health Science. 2008; 18 (2):33-38.

[10] Merid Y, Hegazy M, Mekete G, Teklemariam S. Intestinal helminthic infection among children at Lake Awassa area, south Ethiopia. Ethiopian Journal of Health Development. 2001; 15:31-37. 
[11] Girum T. The prevalence of intestinal helminthic infections and associated risk factors among school children in Babile town, eastern Ethiopia. Ethiopian Journal of Health Development. 2005; 19: 140-147.

[12] Fekadu D, Beyne P, Amha K. Hookworm species distribution among school children in Asendabo Town, Jimma Zone, South West Ethiopia. Ethiopian Journal of Health Science. 2008; 18:53-56.

[13] Mengistu L, Chelsea R, Jones, Sarita K, Berhanu E, Yalemtsehay M. Community's awareness about intestinal schistosomiasis and the prevalence of infection in two endemic localities of Ethiopia. Ethiopian Journal of Health Development. 2009; 19:104.

[14] Birrie H, Tedla S, Tilahun G, Kloos H, Eshete H. Schistosomiasis and its distribution in Ethiopia and Eritrea. In: Schistosomiasis in Ethiopia and Eritrea, Birrie H, Tedla S, Jemaneh L. (Eds.) Institute of Pathobiology, Addis Ababa University Printing Press, 1998, pp.29-86.

[15] Katz N, Chaves A, Pellegrino J. A simple device for quantitative stool thick-smear technique in Schistosoma mansoni. Rev Inst Med Trop Sao Paulo. 1972; 14: 397-400.

[16] Endriss Y, Escher E, Birgit R, Rohr H, Weiss N. Methods in Parasitology; Kato-Katz technique for helminth eggs. 2005, pp13-29.

[17] Brooker S, Moulin A, Luoba D, Bundy M, Kremer D. Epidemiology of Single and Multiple Species of Helminth infections among School Children in Busia District, Kenya. East African Medical Journal. 2000; 77:157-168.

[18] Mutuku A, Mwanthi, Mary K, Kinoti, Annah W, Wamae, et al. Prevalence of intestinal worm infections among primary school children in Nairobi city. Kenya East African Journal of Public Health. 2008; 5(2): 86-89.

[19] Mengistu L, Berhanu E. Prevalence of intestinal parasites among schoolchildren in a rural area close to the southeast of Lake Langano, Ethiopia. Ethiopian Journal of Health Development. 2004; 18:116-120.
[20] Basista R, Yoko O, Ramesh B, Basudha R, Keshav P, Chitra K, et al. Gender variations in the prevalence of parasitic Infections and the level of awareness in adolescents in rural Nepal. Southeast Asian Journal of Tropical Medicine and Public Health. 2001; 32: 575-580.

[21] Fiona M, Fleming, Simon Brooker, Stefan M. Geiger, Iramaya $\mathrm{R}$, et al. Synergistic associations between hookworm and other helminth species in a rural community in Brazil. Tropical Medicine and International Health. 2006; 2:56-64.

[22] Ogwurike B, Ajayi J, Ajayi O, Olaniyan O, Nangna J, Oluwadare A, et al. A Comparative Study of Helminthiasis among Pupils of Private and Public Primary Schools in Jos North Local Government Area of Plateau State, Nigeria. Annals of Natural Sciences; 2010: 28-47.

[23] Kurup R. Control of intestinal parasites among children in two communities of South Saint Lucia. Journal of Rural and Tropical Public Health. 2010; 9:95-100.

[24] Olufemi M, Ndubuisi C, Oluseyi O, Adedayo O, Aliu A, Emmanuel B, et al. Intestinal helminthiases and schistosomiasis among school children in an urban centre and some rural communities in southwest Nigeria. Korean Journal of Parasitology. 2007; 4: 233-238.

[25] Okpala H, Agwu I, Chimezie O, Nwobu G, Ohihoin A. A survey of the prevalence of schistosomiasis among pupils in Apata and Laranto areas in Jos, Plateau State. Online Journal of Health Allied Sciences. 2004; 1:1.

[26] Shewakena F. S.mansoni in Jiga town, Gojam administrative Region. Ethiopian Journal of Health Development. 1995; 9:16.

[27] Rubaihayo J, Moghusu E, Clouds P, Abaasa A. Schistosomiasis transmission at high altitude crater lakes in Western Uganda. BMC Infectious Diseases. 2008; 8:110.

[28] Nkegbe E. Prevalence of schistosomiasis among school children in the lower river Volta basin in Ghana. Gomal Journal of Medical Sciences. 2010; 8 (1):54-56. 